\title{
A SYMBOLIC-NUMERIC METHOD TO ANALYSE HIGH-Q QUARTZ CRYSTAL OSCILLATORS IN FREQUENCY DOMAIN
}

\author{
Nicolas Ratier, Mickaël Bruniaux, Rémi Brendel \\ Institut FEMTO-ST, Département LPMO, \\ 32 avenue de l'Observatoire, 25000 Besançon, France \\ Phone: (33)3818539 99, Fax: (33)3818539 98, e-mail: nicolas.ratier@lpmo.edu
}

\begin{abstract}
We present a symbolic-numeric method dedicated to the simulation of ultra stable quartz oscillators entirely in the frequency domain including the nonlinear parts of the circuit. The main idea is to replace, by symbolic computation, the nonlinear differential system describing the oscillator by a system of nonlinear equations of Fourier coefficients whose solution is an approximation of the steady-state solution. This paper explains how to face the two main difficulties of this symbolic computation: the processing of nonlinear components and the management of large number of coefficients.
\end{abstract}

Keywords: quartz crystal oscillator, simulation, symbolic-numeric.

\section{INTRODUCTION}

The method described here consists in replacing the whole circuit connected to the quartz by a nonlinear dipole whose impedance depends on the current amplitude evaluated at the resonator frequency. It can be shown that the knowledge of this nonlinear dipole is sufficient to determine the oscillator behaviour. Next, the computation of the dipole characteristics is performed in a symbolic way from the circuit equations including the semiconductor elements. Then our symbolic program automatically generates an efficient numerical code to compute the oscillator behaviour.

\section{PRINCIPLE OF THE SYMBOLIC METHOD}

The Colpitts oscillator shown in Fig. 1 is used to illustrate the method described in this paper. The equivalent circuit of the transistor is the regular Ebers-Moll static model [1]. The equivalent circuit of the quartz crystal resonator includes a motional branch $\left(R_{q}, L_{q}, C_{q}\right)$ in parallel with a capacitance $C_{p}$.

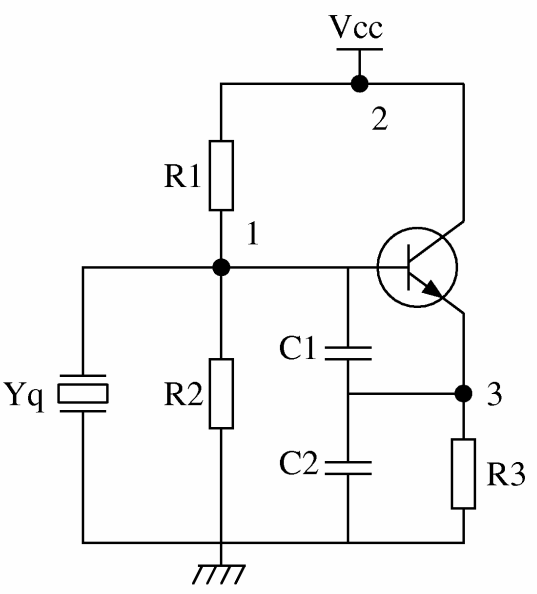

Fig. 1 - Colpitts oscillator

The component values of the circuit and the resonator are given in Table 1. 
Table 1 - Amplifier component and resonator values

\begin{tabular}{|l|c|c|c|c|c|c|c|}
\hline$R_{1}$ & $36800 \Omega$ & $R_{3}$ & $1769,2 \Omega$ & $C_{1}$ & $151,65 p F$ & $f_{q}$ & $5 \mathrm{MHz}$ \\
\hline$R_{2}$ & $35290 \Omega$ & $V c c$ & $15 V$ & $C_{2}$ & $148 p F$ & $R_{q}$ & $670 \Omega$ \\
\hline
\end{tabular}

The motional branch of the quartz is replaced by a sinusoidal current source of amplitude $a$ and frequency $f_{q}$. The current source allows one to defined the nonlinear dipole $R_{d}(a)$ and $L_{d}(a)$, both functions of the loop current amplitude $a$. The knowledge of $R d(a)$ and $\operatorname{Ld}(a)$ is sufficient to determine the behaviour of the oscillator [2].

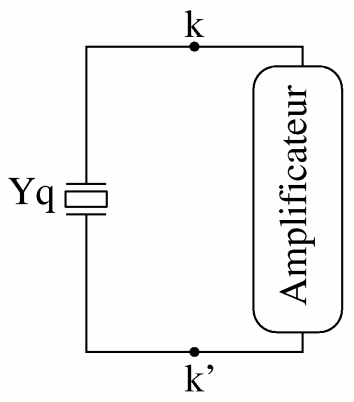

(a)

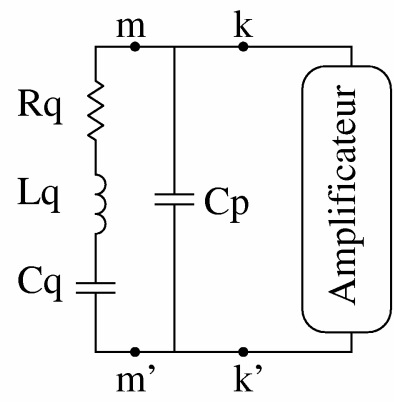

(b)

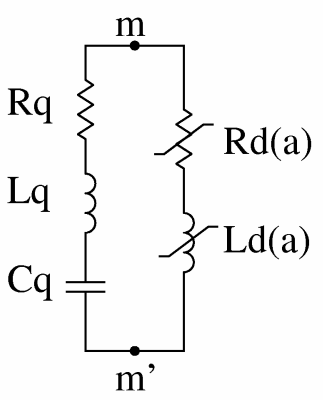

(c)

Fig. 2 - Dipolar representation of the oscillator

The system of equations that models the electronic circuit is done symbolically by the Modified Nodal Method (MNM) [3]. If the circuit contains $n$ nodes and $n_{v}$ voltage-defined branches, then the $n-1$ nondatum node voltages and the $n_{v}$ voltage-defined branch currents become the unknown circuit variables. The set of modified nodal equations are obtained by applying the KCL (Kirchhoff's Current Law) to each nondatum node and including the $n_{v}$ voltage-defined branch relations. That leads to the algebraic-differential system (1)-(4).

$$
\begin{gathered}
-G_{1}\left(V_{2}(t)-V_{1}(t)\right)+G_{2} V_{1}(t)+C_{1}\left(\frac{\partial V_{1}(t)}{\partial t}\right)-C_{1}\left(\frac{\partial V_{3}(t)}{\partial t}\right)-\frac{I_{S}}{\beta_{R}} \exp \left(\frac{V_{1}(t)-V_{3}(t)}{U_{T}}\right) \\
-\frac{I_{S}}{\beta_{F}} \exp \left(\frac{V_{1}(t)-V_{2}(t)}{U_{T}}\right)+a \sin (\omega \mathrm{t})=0 \\
I_{1}(t)+G_{1}\left(V_{2}(t)-V_{1}(t)\right)+\frac{I_{S}}{\beta_{R}}\left(\exp \left(\frac{V_{1}(t)-V_{2}(t)}{U_{T}}\right)-1\right)+\frac{I_{S}}{\beta_{F}} \exp \left(\frac{V_{2}(t)-V_{3}(t)}{U_{T}}\right)=0 \\
G_{3} V_{3}(t)+G_{2} V_{1}(t)+C_{1}\left(\frac{\partial V_{1}(t)}{\partial t}\right)-C_{1}\left(\frac{\partial V_{3}(t)}{\partial t}\right)+C_{2}\left(\frac{\partial V_{3}(t)}{\partial t}\right)+\frac{I_{S}}{\beta_{R}} \exp \left(\frac{V_{1}(t)-V_{3}(t)}{U_{T}}\right) \\
+\frac{I_{S}}{\beta_{F}} \exp \left(\frac{V_{2}(t)-V_{3}(t)}{U_{T}}\right)=0 \\
V_{2}(t)-V C C=0
\end{gathered}
$$

The sinusoidal current source ensures periodic signals in the whole circuit, so we assume a solution, for the unknowns $V_{l}(t), V_{2}(t), V_{3}(t)$ and $I_{l}(t)$, of the following form where $I_{l}(t)$ is the current trough the voltage generator $V c c$.

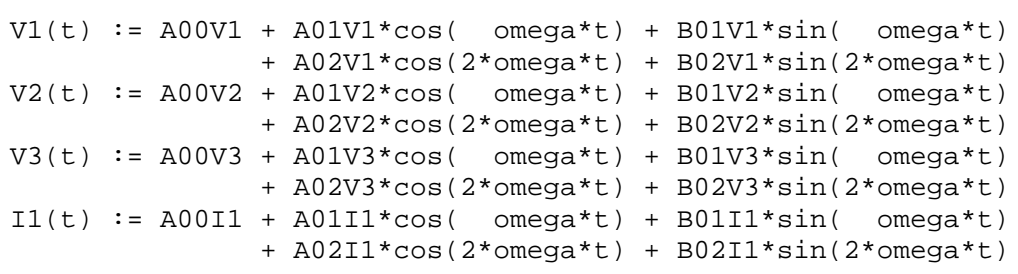


By substituting the assumed solution and its derivative into the given differential system (1)-(4), it is possible to write the system under the following form where the parameters TT229, ..., TT572 are all functions of the previous A००V1, .., BO2I1.

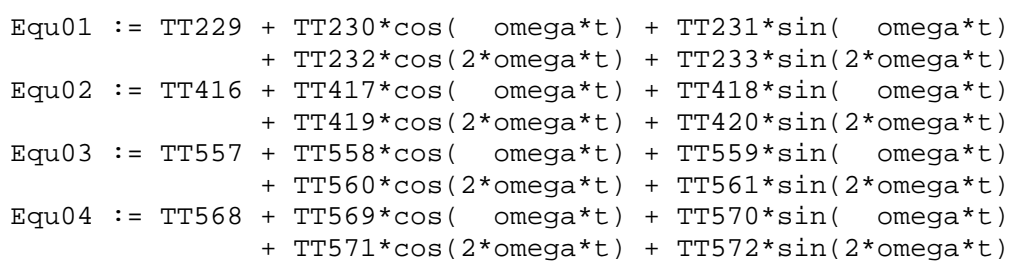

The amplitude $a$ of the sinusoidal current source is a parameter of the system. A simple calculation shows that $R_{d}(a)$ and $L_{d}(a)$ can be obtained as shown in (5) and (6).

$$
\begin{aligned}
R_{d}(a) & =\frac{B 01 V 1}{a} \\
L_{q}(a) & =\frac{A 01 V 1}{a \omega_{q}}
\end{aligned}
$$

The steady state amplitude $a_{0}$ is obtained by adding the nonlinear equation (7) to the differential system (1)-(4).

$$
R_{q}+R_{d}(a)=0
$$

The steady state frequency $\omega_{0}$ of the oscillation is given by Eq. (8).

$$
\omega_{0}^{2}=\omega_{q}^{2}\left(1-\frac{L_{d}\left(a_{0}\right)}{L_{q}}\right)
$$

\section{HOW TO ACHIEVE A LINEAR COMPLEXITY?}

A direct and naive application of the method to build the nonlinear system leads to an exponential growth of the term number in the expressions of TT229, ..., TT572. A second problem is the reduction into a series form of the exponential functions.

A solution to the problem of the exponential growth of the term number is to replace in a first step each differential equation by a binary tree, (for instance, the binary tree of Eq. (3) is shown in Fig. (3)), then to parse the tree to progressively reduce it under a series form from the bottom to the top.

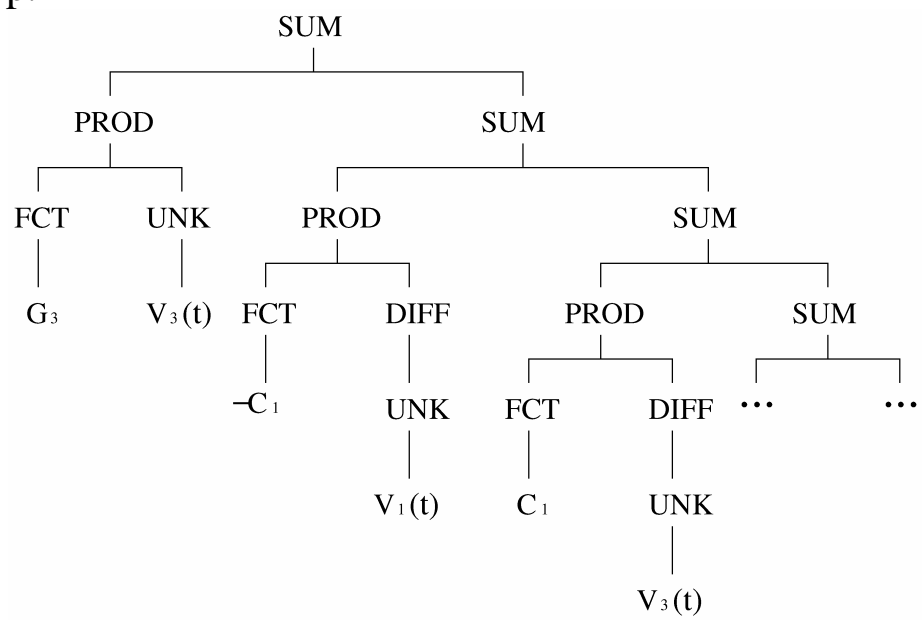

Fig. 3 - Binary tree of Eq. (3)

At the beginning, all the leaves are under a series form. During the reduction process, at each node new coefficients expressed in function of the previous ones will be generated. At the final step, only a Fourier series with manageable coefficients will remain. 
The management of the exponential function is quite similar to the previous one. It is based on the construction of a binary tree and the generation of new coefficients in function of the previous ones during the tree parsing. Here, The construction of the tree is based on the addition formulae of exponential. As an example, the binary tree for exponential function is shown in Fig. (4). Of course, each transcendental function will have its own associated tree.

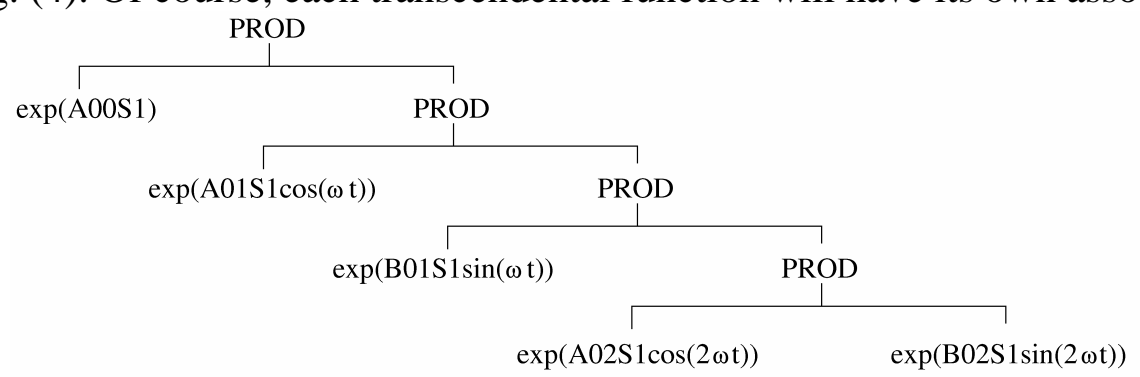

Fig. 4 - Binary tree of exponential function

The reduction of the nodes has been previously explained. The reduction of the leaves is based on the associated Bessel series expansion of exponential function (9) and (10) where $I_{k}(z)$ are the Bessel functions of the second kind and of integer order.

$$
\begin{gathered}
\exp (z \cdot \cos \theta)=I_{0}(z)+2 \sum_{k=1}^{\infty} I_{k}(z) \cos (k \theta) \\
\exp (z \cdot \sin \theta)=I_{0}(z)+2 \sum_{k=1}^{\infty}(-1)^{k} I_{2 k}(z) \cos (2 k \theta)+2 \sum_{k=1}^{\infty}(-1)^{k} I_{2 k+1}(z) \sin ((2 k+1) \theta)
\end{gathered}
$$

Once the differential equation system is transformed into a sparse nonlinear equation system of the Fourier coefficient of the unknowns, it can be solved by numerical method. The solution for the Colpitts oscillator studied is given in Table 2.

\begin{tabular}{|c|c|c|c|c|c|}
\hline & $A \odot \odot$ & $A 01$ & B01 & $A \odot 2$ & $\mathrm{~B} 02$ \\
\hline$V_{1}(t)$ & 4.948 & 0.887 & 0.948 & 0.055 & 0.512 \\
\hline$V_{2}(t)$ & 15 & $-1.327 \mathrm{e}-15$ & $-6.229 e-16$ & $-1.046 \mathrm{e}-15$ & $-1.351 \mathrm{e}-15$ \\
\hline$V_{3}(t)$ & 4.669 & 0.524 & 1.008 & 0.053 & 0.537 \\
\hline$I_{1}(t)$ & $-0.277 \mathrm{e}-3$ & $1.519 \mathrm{e}-5$ & $2.756 e-5$ & $-6.620 \mathrm{e}-6$ & $1.730 \mathrm{e}-5$ \\
\hline
\end{tabular}

Table 2 - Fourier coefficients of the unknowns

\section{CONCLUSION}

A solution based on tree parsing to manage the large number of coefficients inherent to symbolic computation has been proposed. Unlike all other harmonic methods, the linear and the nonlinear parts of the differential equation of the circuit are processed in an uniform way in the Fourier domain. The numerical simulation times of the equations generated in the last step of this method is independent on the circuit time constants, because our harmonic method imposes the steady-state conditions by virtue of Fourier expansion of the unknowns.

\section{REFERENCES}

1. G. Massobrio and P. Antognetti, Semiconductor Device Modeling with SPICE, MacGrawHill, Inc, second edition, 1993.

2. M. Addouche, R. Brendel, D. Gillet, N. Ratier, "Modeling of Quartz Crystal Oscillators by Using Nonlinear Dipolar Method”, IEEE. Trans. On Ultrasonics, Ferroelectrics and Frequency Control, vol. 50, n5, May 2003.

3. J. Vlach and K. Singhal. Computer methods for circuit analysis and design, Van Nostrand Reinhold, 1994. 